\title{
Plasmon-Coupled Photocapacitor Neuromodulators
}

\author{
Rustamzhon Melikov, Shashi Bhushan Srivastava, Onuralp Karatum, Itir Bakis Dogru-Yuksel, \\ Houman Bahmani Jalali, Sadra Sadeghi, Ugur Meric Dikbas, Burak Ulgut, Ibrahim Halil Kavakli, \\ Arif E. Cetin, and Sedat Nizamoglu*
}

Cite This: ACS Appl. Mater. Interfaces 2020, 12, 35940-35949

Read Online

ACCESS | 岁 Metrics \& More | 回 Article Recommendations | St Supporting Information
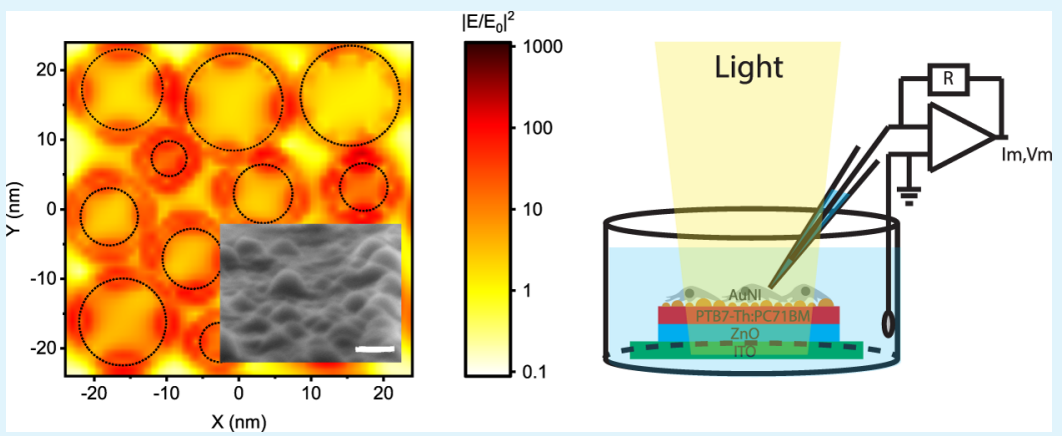

ABSTRACT: Efficient transduction of optical energy to bioelectrical stimuli is an important goal for effective communication with biological systems. For that, plasmonics has a significant potential via boosting the light-matter interactions. However, plasmonics has been primarily used for heat-induced cell stimulation due to membrane capacitance change (i.e., optocapacitance). Instead, here, we demonstrate that plasmonic coupling to photocapacitor biointerfaces improves safe and efficacious neuromodulating displacement charges for an average of $185 \%$ in the entire visible spectrum while maintaining the faradic currents below $1 \%$. Hotelectron injection dominantly leads the enhancement of displacement current in the blue spectral window, and the nanoantenna effect is mainly responsible for the improvement in the red spectral region. The plasmonic photocapacitor facilitates wireless modulation of single cells at three orders of magnitude below the maximum retinal intensity levels, corresponding to one of the most sensitive optoelectronic neural interfaces. This study introduces a new way of using plasmonics for safe and effective photostimulation of neurons and paves the way toward ultrasensitive plasmon-assisted neurostimulation devices.

KEYWORDS: plasmonics, biointerface, photostimulation, photocapacitor, nanoislands, charge transfer, organic polymers

\section{INTRODUCTION}

Extracellular stimulation is the basis for communication with the biological systems to recover lost functionalities, ${ }^{1}$ understand cellular processes, ${ }^{2}$ and switch neural networks. ${ }^{3}$ For that, light offers a noninvasive neuromodulation trigger with high spatial and temporal precision. ${ }^{4-8}$ Optogenetics introduces light-sensitive opsins into the membrane via a viral transfection, but genetic modification currently raises concerns about their clinical use. ${ }^{9,10}$ Alternatively, geneless optical stimulation of cells has a high potential to control the neural activity. ${ }^{11,12}$ For that, optoelectronic biointerfaces can generate photoexcited electrons and holes that can be manipulated for faradic, capacitive, and thermal photostimulation of neurons.

Plasmonics has a high potential to increase the performance of optoelectronic biointerfaces. In principle, plasmonic nanostructures can concentrate the incoming radiation to a subwavelength spatial profile due to localized surface plasmon resonance (LSPR) ${ }^{13}$ and may boost the light-matter interactions for sensitive transduction of optical signals to bioelectrical stimuli. In addition, the light-induced plasmon energy can be transferred to the conduction band of the nanostructure that can produce energetic electrons, known as hot electrons, and these charges may be controlled for photostimulation of neurons. However, despite its high potential for extracellular stimulation, plasmonics has been mainly used for photothermal transmembrane modulation of neurons. ${ }^{14-18}$ Light is converted to heat energy due to decay of plasmon oscillations, and the resultant temperature variation induces a membrane potential change through a transient membrane capacitance shift. ${ }^{14}$ Recently, some pieces of evidence showing that metal decoration can support an increase of capacitive and faradic currents were also reported. ${ }^{8}$

Received: May 23, 2020

Accepted: July 15, 2020

Published: July 15, 2020 
(a)
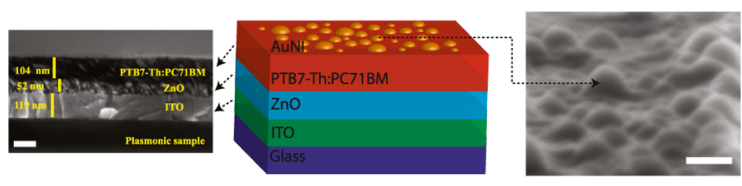

(b)

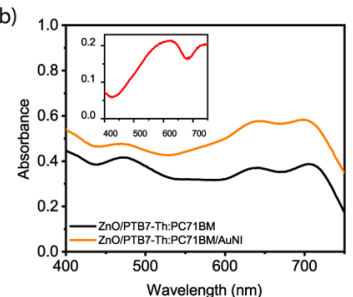

(d)

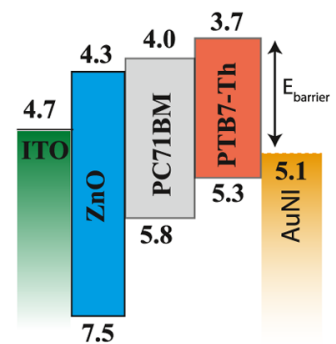

(c)
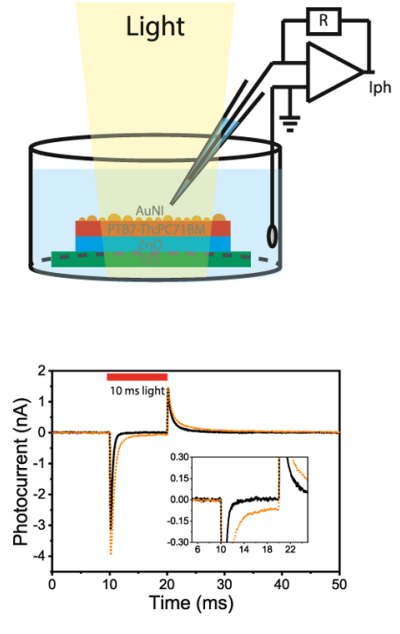
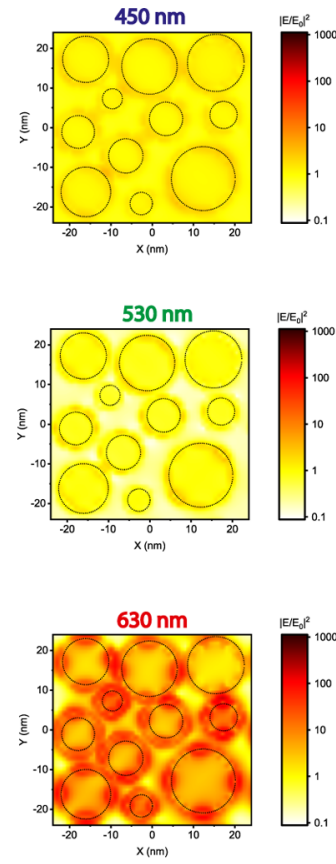

Figure 1. (a) (Left) Cross-sectional scanning electron microscopy (SEM) image of the plasmonic biointerface (scale bar, 100 nm). (Middle) Schematic device structure of the plasmonic (ITO/ZnO/PTB7-Th:PC71BM/AuNI) biointerface. (Right) SEM image of nanoislands at the plasmonic biointerface (scale bar, $20 \mathrm{~nm}$ ). (b) Absorption spectrum of ZnO/PTB7-Th:PC71BM (black line) and ZnO/PTB7-Th:PC71BM/AuNI (orange line) thin films. The inset shows absorbance of the AuNI layer (red line), which is calculated by extracting it from the control biointerface without the gold layer that has a smooth surface due to the absence of nanoislands (Figure S1a,b). (c) Schematic of the photocurrent measurement setup. The patch pipette is kept close to the surface, and the current is measured with a voltage-clamp mode. (d) Energy band diagram of the plasmonic biointerface (with respect to vacuum). (e) Photocurrent of control (black line) and plasmonic (orange dotted line) biointerfaces illuminated under green light at $8.8 \pm 0.2 \times 10^{16}$ photons $\mathrm{s}^{-1} \cdot \mathrm{cm}^{-2}$ with $10 \mathrm{~ms}$ pulse widths. The inset zooms on low photocurrent levels. The capacitive current amplitude is defined as the maximal current amplitude reached after the light onset. The faradic current is defined as the current amplitude for $9 \mathrm{~ms}$ after the start of illumination (Figure S5). (f) Lumerical FDTD simulation of AuNI of the plasmonic biointerface. Top, middle, and bottom panels show field profiles at 450,530 , and $630 \mathrm{~nm}$, respectively. $\mathrm{KE}_{\text {electron }}=E_{\text {photon }}-E_{\text {barrier }}-E_{\text {deep traps }}, E_{\text {barrier }}=1.4 \mathrm{eV}, E_{\text {deep trap }}=$ $[0.5-0.8] \mathrm{eV} . \mathrm{KE}_{\text {electron at }} 450 \mathrm{~nm}=2.75-1.4-0.8=+0.55 \mathrm{eV}, \mathrm{KE}_{\text {electron at }} 530 \mathrm{~nm}=+0.14 \mathrm{eV}$ and $\mathrm{KE}_{\text {electron at } 630 \mathrm{~nm}}=-0.23 \mathrm{eV}$. While there is hotelectron injection in blue and green spectral regions, we observe the nanoantenna effect at the red spectral region.

In this study, we demonstrate that the coupling of plasmons to photocapacitors can improve the charge injection of the neuromodulating displacement currents in the entire visible range. We proved that this broadband enhancement ability mainly stems from hot-electron injection in blue and the local field enhancement in red spectral regions. Finally, the plasmonassisted capacitive photocurrent modulates transmembrane potential at a single-cell level.

\section{RESULTS}

Structure of the Plasmonic Biointerface. Capacitive charge injection, which is a safe method for stimulation of neurons, ${ }^{8}$ is based on the electromagnetic attraction and repulsion of the ions in the biological medium due to charge movement in the stimulating electrode. For that, the fabrication of the plasmonic biointerface architecture (Figure 1a) started with the sequential deposition of the $\mathrm{ZnO}$ and bulk heterojunction composite PTB7-Th:PC71BM layers on the indium tin oxide (ITO) substrate. According to cross-sectional scanning electron microscopy (SEM) (Figure 1a, left), the thicknesses of the $\mathrm{ZnO}$ and PTB7-Th:PC71BM layers correspond to 52 and $104 \mathrm{~nm}$, respectively. Afterward, the gold $(\mathrm{Au})$ thin film is deposited and annealed to form selfassembled nanoislands. ${ }^{19,20}$ The mechanism of island formation is described by Ostwald ripening of mass transport either by weakly bound individual Au atoms or small clusters combining into larger clusters. ${ }^{21}$ The surface morphology of the plasmonic biointerface confirms the formation of gold nanoislands (AuNIs) on the photoactive layer (Figure 1a, right), which leads to a plasmonic peak at around $626 \mathrm{~nm}$ (Figure $1 \mathrm{~b}$, inset). The broad plasmonic band stems from low annealing temperatures, ${ }^{19}$ which is required to retain optical properties of the organic photoactive layer. The absorption band of the plasmonic biointerface covers all of the visible range, which is useful for the conversion of light within the entire visible range to bioelectrical stimuli (Figure $1 b$ ).

Photocurrent Analysis. We investigate the photocurrent response of the plasmonic biointerface in an artificial cerebrospinal fluid (aCSF) solution. We use a patch-clamp system (HEKA, EPC 800) in voltage-clamp mode (with $\sim 6$ $\mathrm{M} \Omega$ patch pipette tips) under the free-standing condition (Figure 1c). Since the optoelectronic processes are quantal, the biointerfaces were pumped under illumination of blue, green, and red lights by keeping the photon counts at the same level (Figures S2 and S3), which correspond to $8.8 \pm 0.2 \times 10^{16}$ photons $\mathrm{s}^{-1} \cdot \mathrm{cm}^{-2}$ with $10 \mathrm{~ms}$ pulse widths. The plasmonic biointerface generates several nanoamperes of photocurrent in aCSF in the entire visible spectrum (Figure S4). Among different excitation wavelengths, the green spectral window shows the highest photocurrent for both biointerfaces due to 
(a)

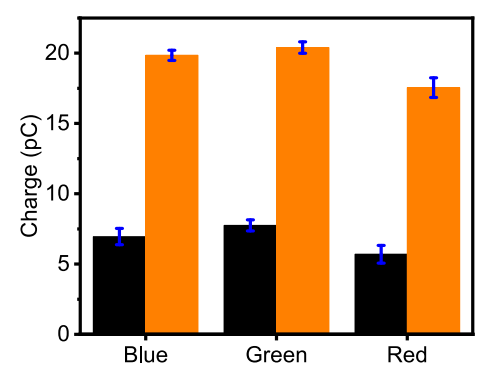

(c)

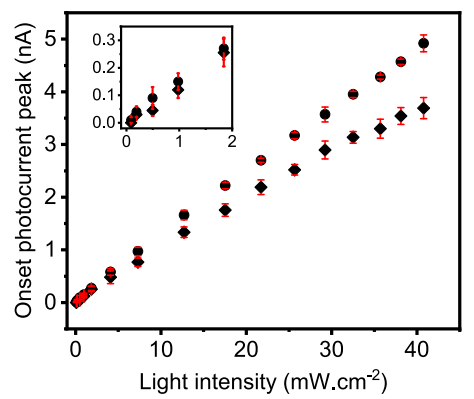

(b)

(d)
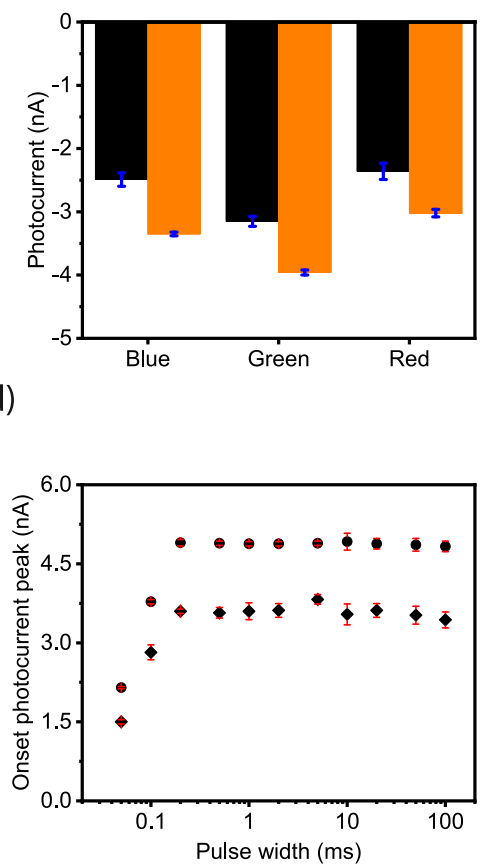

Figure 2. (a) Injected amount of charges and (b) current peaks of the control (black) and plasmonic biointerfaces (orange) at $8.8 \pm 0.2 \times 10^{16}$ photons $\mathrm{s}^{-1} \cdot \mathrm{cm}^{-2}$ with $10 \mathrm{~ms}$ pulse widths. (c) Magnitude of the peak of capacitive photocurrentcurrents of the control (rhombus dots) and plasmonic (circle dots) biointerfaces under different light intensity levels. (d) Magnitude of the peak capacitive currents generated by the control (rhombus dots) and plasmonic (circle dots) biointerfaces under different pulse widths having light intensity at $8.8 \pm 0.2 \times 10^{16} \mathrm{photons} \mathrm{s}^{-1} \cdot \mathrm{cm}^{-2}$.

higher absorption strength and external quantum efficiency of the photoactive blend ${ }^{22}$ in comparison with blue and red excitation (Figure S4). Two spikes were observed at the onset and offset of light illumination. These two spikes (i.e., onset and offset peaks) show that the charge-transfer mechanism is based on the capacitive charge transfer. ${ }^{23}$

Here, the current generation mechanism is controlled by the energy band alignment of the layers at the biointerface (Figure 1d). Photogenerated charge carriers initially dissociate in the photoactive blend, and electron transport toward the $\mathrm{ZnO}$ layer takes place. Holes are mainly localized in the proximity of the electrolyte interface that induces a displacement current. Hence, the initial hole accumulation near the electrolyte interface generates onset capacitive photocurrent from the biointerface to the electrolyte (Figure 1e). After the light is turned off, the decrease of the hole concentration due to recombination leads to another offset capacitive spike in the opposite direction.

We compared the light-induced current and charge generation characteristics of the biointerface with those of the control biointerface. For the analysis, we identified faradic and capacitive contributions in blue, green, and red spectral windows under the same photon counts (Figure S5 and eqs S1-S4). The total capacitive charge transfer to the electrolyte is improved by $185.6,163.2$, and $207.9 \%$ in blue, green, and red spectral windows, respectively, which correspond to an average increase of $185 \%$ in the entire visible spectrum (Figure $2 \mathrm{a}$ and Tables S1 and S2). Furthermore, the plasmonic biointerface enhanced the peak photocurrents to the levels of $34.5,28.0$, and $25.7 \%$ for blue, green, and red illumination, respectively (Figure $2 \mathrm{~b}$ and Tables S3 and S4). We observed that since the lowest unoccupied molecular orbital (LUMO) energy of PTB7-Th is above the water oxidation energy, the faradic current due to hole transfer to electrolyte is significantly suppressed, which corresponds to less than $1 \%$ of the total photocurrent (Figure S6). The linear response of the biointerface to the light intensity indicates a single-photonabsorption-induced charge carrier generation (Figures $2 \mathrm{c}$ and S7). We also analyzed the possible contribution by the photothermal effects, and since the intensity levels that the plasmonic biointerface operates were several orders of magnitude lower than the heat-induced neurostimulation, we did not observe any temperature variation in the solution even under $170 \mathrm{~mW} \cdot \mathrm{cm}^{-2}$ (Figure S8). Furthermore, we varied the pulse width between $50 \mu \mathrm{s}$ and $100 \mathrm{~ms}$ and observed that the peak capacitive currents are well maintained for the pulses longer than $200 \mu \mathrm{s}$, which are sufficiently short to elicit targeted electrophysiological processes. The decrease of the photocurrent amplitude below $200 \mu$ s stems from the strong overlap between the onset and offset peaks of the capacitive spikes (Figures 2d and S6).

As a result of the simulations, we did not observe any nanoantenna effect in green and blue spectral regions (Figure 1f). For example, when photons are absorbed by AuNI, electrons gain kinetic energy, which can be calculated as follows: $\mathrm{KE}_{\text {electron }}=E_{\text {photon }}-E_{\text {barrier }}-E_{\text {deep traps }}$, where $E_{\text {barrier }}=$ $1.4 \mathrm{eV}$ and $E_{\text {deep trap }}=[0.5-0.8] \mathrm{eV}$. Assuming the highest energy deep trap states, under blue excitation, the kinetic energy of the electron is $\mathrm{KE}_{\text {electron at } 450 \mathrm{~nm}}=2.75-1.4-0.8=$ $+0.55 \mathrm{eV}$. Hence, hot-electron injection is possible. When the plasmonic biointerface is illuminated with a green lightemitting diode (LED) at $530 \mathrm{~nm}$, the kinetic energy of the electron $\mathrm{KE}_{\text {electron at } 530 \mathrm{~nm}}=+0.14 \mathrm{eV}$ is enough to transport it to the photoactive layer. Hence, under green excitation, hotelectron injection is also probable. Finally, under red LED excitation at $630 \mathrm{~nm}$, the kinetic energy of the electrons is 
(a)

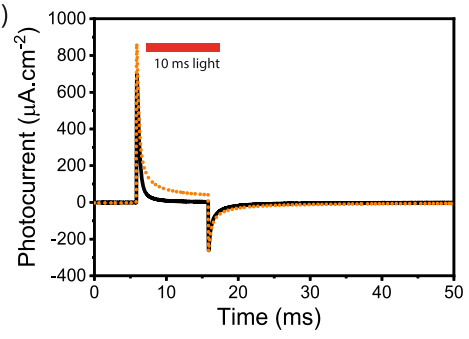

(d)

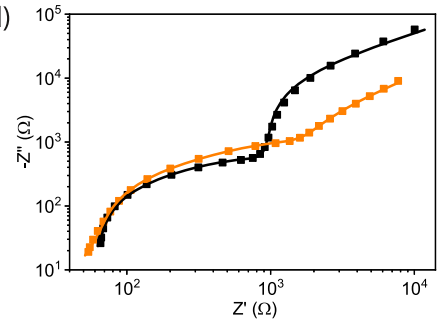

(b)

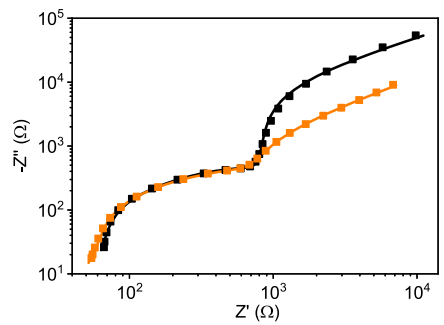

(e)

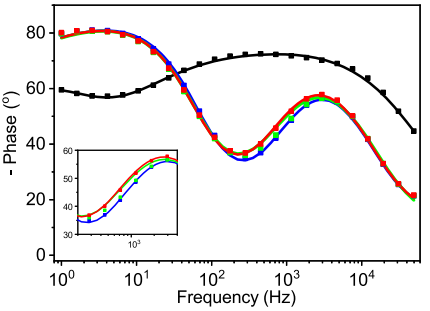

(c)

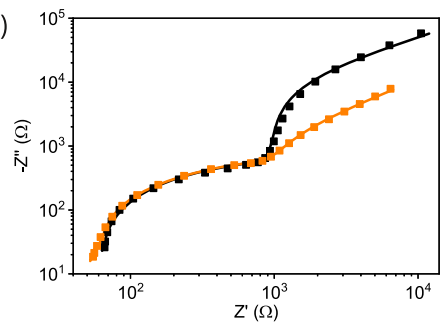

(f)

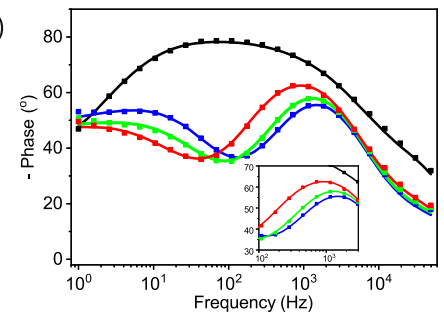

(g)

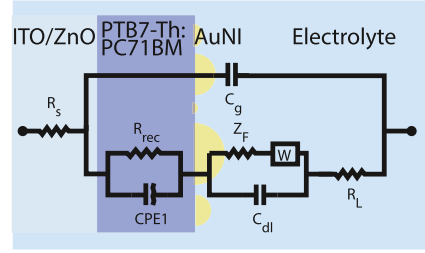

Figure 3. Electrochemical characterization of the control and plasmonic biointerfaces. (a) Photocurrent of control (black line) and plasmonic (orange dotted line) biointerfaces measured by the two-probe electrochemical setup, where the working electrode is connected to ITO and counter and reference electrodes are in the extracellular solution. Nyquist plots of the control (black) and plasmonic biointerfaces (orange) (b) under blue, (c) green, and (d) red excitation. Bode phases of (e) control and (f) plasmonic biointerfaces measured by the three-probe electrochemical setup. The inset zooms between 50 and $5000 \mathrm{~Hz}$. (g) Circuit diagram fitting the Nyquist and Bode plots for both the control and plasmonic biointerfaces.

$\mathrm{KE}_{\text {electron at } 630 \mathrm{~nm}}=-0.23 \mathrm{eV}$. Hence, hot-electron injection in the red spectral region is unlikely. In summary, there is a dominant hot-electron injection in blue and green spectral regions and we observe the nanoantenna effect in the red spectral region.

Electrochemical Investigation into Plasmonic Enhancement. We investigate the enhancement of the displacement current by the plasmonic biointerface using electrochemical impedance spectroscopy. For that, we did threeprobe measurements in aCSF media using $\mathrm{Ag} / \mathrm{AgCl}$ as a reference electrode, platinum as a counter electrode, and the biointerface as the working electrode. ${ }^{24}$ To verify enhancement in the electrophysiology setup, initially, we measured the photocurrent levels and observed a current level of $862 \mu \mathrm{A}$. $\mathrm{cm}^{-2}$ that inject $1.2 \mu \mathrm{C} \cdot \mathrm{cm}^{-2}$ under $50 \mathrm{~mW} \cdot \mathrm{cm}^{-2}$ blue LED excitation for the plasmonic photoelectrode. Hence, with respect to the control group, the capacitive charge transfer and peak photocurrent were improved by 182 and 24\%, respectively, which is similar to the enhancement observed by the electrophysiology setup (Figure 3a). The physical mechanisms at the various interfaces are probed in response to a small AC perturbation of $10 \mathrm{mV}\left(V_{\text {rms }}\right)$ varied over a frequency range from $1 \mathrm{~Hz}$ to $0.1 \mathrm{MHz}$ at zero $\mathrm{DC}$ bias. Impedance responses of the control and plasmonic biointerfaces are semicircle in the high- to mid-frequency region and a depressed semicircle with a linear extension in the lowfrequency region in the complex plane (Figure $3 b-d$ ). The Bode plot at high frequency is generally interpreted as the electrolyte region and in the $\mathrm{kHz}$ frequency region corresponds to the double layer formed at the photoelectrode/electrolyte interface (Figure S9). The Bode phase angle $\left(\sim 56^{\circ}\right)$ is found similar for both biointerfaces under blue and green light illumination, and it is interestingly observed higher $\left(\sim 62^{\circ}\right)$ for the plasmonic biointerface under red light illumination (Figure $3 e, f)$.

To correlate with the physical mechanisms in the plasmonic biointerface, we analyzed the frequency response and fitted the equivalent circuit model $\left(\chi^{2}=0.01-0.03\right)$ comprising a metal-insulator-metal structure (Figure $3 \mathrm{~g}$ ). $C_{\mathrm{g}}$ represents the geometrical capacitance of the device, and $R_{\mathrm{s}}$ is assigned to the electrical resistance that is present at metal electrodes and electrode/contact layer/active layer interfaces. The recombination resistance $\left(R_{\text {rec }}\right)$ in parallel with the constant phase element (CPE1) corresponds to the charge dynamics in the photoactive bulk heterojunction. The random characteristics of the blend active layer in the organic bulk heterojunction can be attributed to the distribution of relaxation times and are related to the impedance as $Z=Y_{0}^{-1}(j \omega)^{-n}$, where $Y_{0}$ is the CPE coefficient and the ideality factor $n-0$ for pure resistive and 1 for pure capacitive behavior-is the characteristic of the distribution of relaxation times. ${ }^{25}$ The plasmonic photoelectrode/electrolyte interface is defined by the parallel combination of the Warburg element $(W)$ with the doublelayer capacitance $\left(C_{\mathrm{dl}}\right)$. Using the fitted macroelectrical parameters (Table S5), the optoelectronic parameters of the biointerfaces such as the effective lifetime $\left(\tau_{\mathrm{n}}\right)$ and mobility $\left(\mu_{\mathrm{n}}\right)$ of charge carriers are determined (Table S6).

We observed that while the recombination resistance $\left(R_{\text {rec }}\right)$ is similar for both control and plasmonic biointerfaces under blue and green light illumination, it increases approximately by two times $\left(R_{\mathrm{rec}}=1.6 \mathrm{k} \Omega\right)$ for the plasmonic biointerface under red light illumination in comparison with that for the control 
(a)

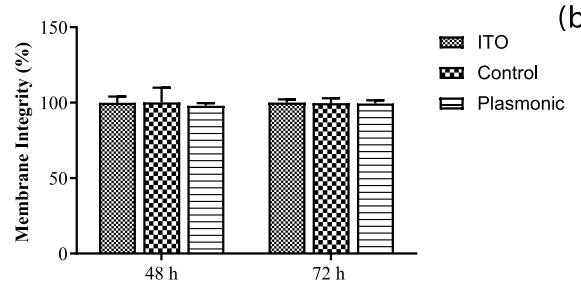

(c)

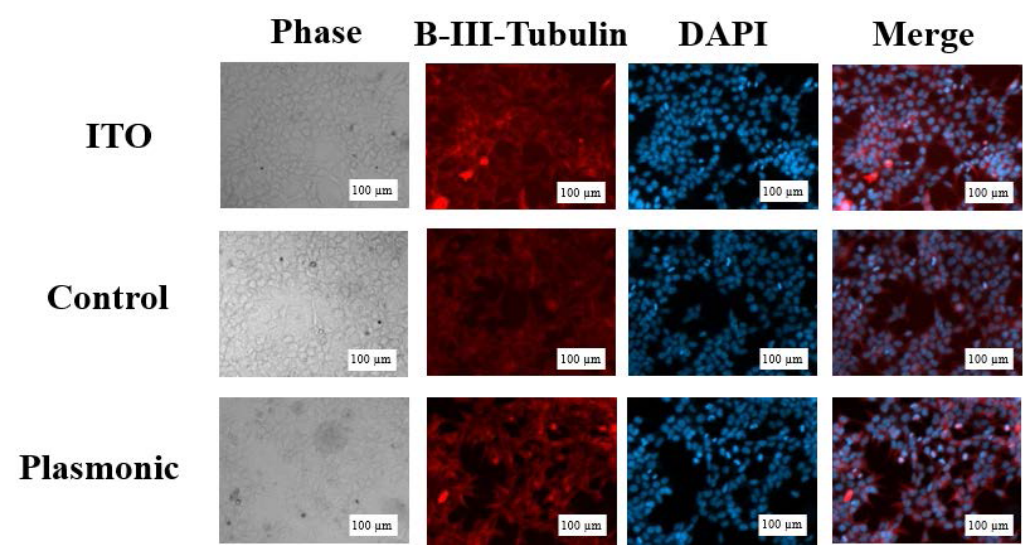

(b)

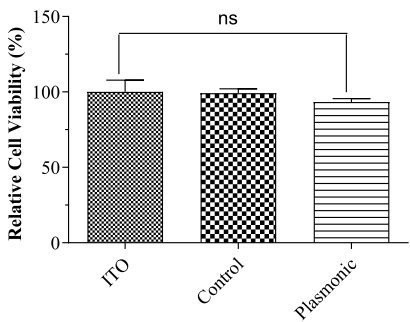

Figure 4. (a) MTT assay, assessment of the effect of plasmonic biointerfaces on mitochondrial activity of SHSY-5Y cells. Cell viability on biointerfaces was presented relative to the ITO control. Results are presented in a column graph plotting the mean with standard error of the mean $(\mathrm{SEM})$. Experiments were performed with at least three technical replicates and repeated three times $(n=3)$. An unpaired two-tailed $t$-test was performed to determine the level of significance. ${ }^{*} p<0.05$ was considered as statistically significant; nonsignificant differences are presented as "ns." (b) LDH leakage assay, assessment of membrane integrity of the cells grown on biointerfaces. Experiments were performed with at least three technical replicates and repeated three times $(n=3)$. (c) Immunofluorescence imaging, the effect of biointerfaces on the morphology of SHSY-5Y cells. The morphology of the cells grown on biointerfaces was visualized by a fluorescence microscope after $\beta$-III tubulin immunolabeling and DAPI staining (scale bar, $100 \mu \mathrm{m}$ ).

biointerface. This leads to a rise of effective recombination lifetime and indicates the generation of a large number of photoactivated charge carriers. Since the peak plasmonic absorption by the gold nanoislands is in the red spectral range, the presence of gold nanoislands at the biointerface can increase the electron concentration by near-field enhancement of the incoming electromagnetic wave, which is observed by an increase in the corresponding recombination lifetime (Table S6).

In the blue spectral region, while the recombination resistance $\left(\mathrm{R}_{\mathrm{rec}}=0.66 \mathrm{k} \Omega\right)$ and effective lifetime $\left(\tau_{\mathrm{n}}=0.3\right.$ $\mathrm{ms}$ ) remain at a similar level to those of the control biointerface, there is an increase in electron mobility for blue excitation compared to red excitation of the plasmonic biointerface. This means that electron injection becomes more probable in the blue spectral range. According to the band energy diagram, the energy of blue photons can lead to the excitation of the electrons to an energy level that is higher than the barrier between gold nanoislands and the highest occupied molecular orbital (HOMO) level of donor molecules (PTB7-Th). Hence, the hot electrons with high kinetic energy have a higher probability to reach the ITO/ $\mathrm{ZnO}$ interface. ${ }^{26}$ This shows that while in the red spectral region the nanoantenna effect is dominantly responsible for near-field enhancement, in the blue spectral range, hot carrier injection becomes the leading factor. Furthermore, the increase of the double-layer capacitance at all wavelengths from 2.1 to $3.1 \mu \mathrm{F}$ $\mathrm{cm}^{-2}$ supports the plasmonic enhancement of capacitive photocurrent under each color.
Biocompatibility. To test the biological and electrophysiological activity, we used SHSY-5Y cells. These kinds of nonspiking cells such as $\mathrm{N} 2 \mathrm{~A}$ and oocytes, are already used to prove the neuromodulation ability. ${ }^{27,28}$ Initially, we tested the mitochondrial activity with 3-(4,5-dimethylthiazol-2-yl)-2,5diphenyltetrazolium bromide (MTT) and lactate dehydrogenase $(\mathrm{LDH})$ assays to determine the toxicity of the plasmonic biointerfaces on the SHSY-5Y cell line. In these assays, we used the ITO substrate as the control group. Plasmonic biointerfaces did not exhibit any toxicity on the cells by either assay (Figure 4a,b). We also evaluated the effect of plasmonic biointerfaces on cell growth by investigating the morphology of the SH-SY5Y cells via fluorescence microscopy. Cells grown on the samples were fixed with $4 \%$ paraformaldehyde (PFA). Nuclei of the cells were stained with 4',6-diamidino-2phenylindole (DAPI), and the cytoplasm was visualized by anti- $\beta$ III tubulin immunolabeling. As can be seen in Figure $4 c$, cells grown on biointerfaces exhibited comparable morphology with the cells grown on the ITO control. All of these results suggested that plasmonic biointerfaces are suitable for biological experiments with SHSY-5Y cells.

Photostimulation of Neurons. To investigate the membrane potential variation, we perform patch clamping in a whole-cell configuration (Figure 5a). The $I-V$ characteristics of SHSY-5Y cells grown on both control and plasmonic biointerfaces under the dark condition shows that the SHSY$5 Y$ cells exhibit typical resting membrane potential in the range between -20 and $-40 \mathrm{mV}$ (Figure 5b). The SHSY-5Y cells exhibit a quasi-linear response around the resting membrane 
(a)

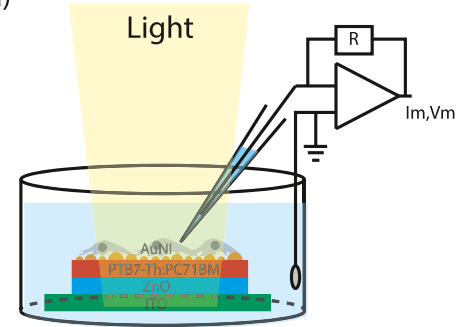

(d)

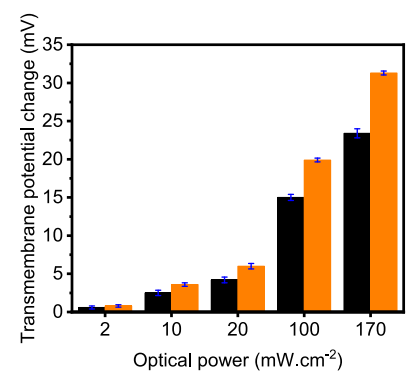

(g)

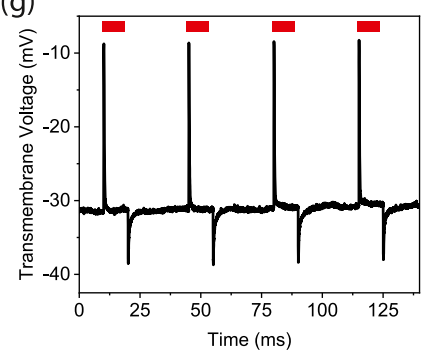

(b)

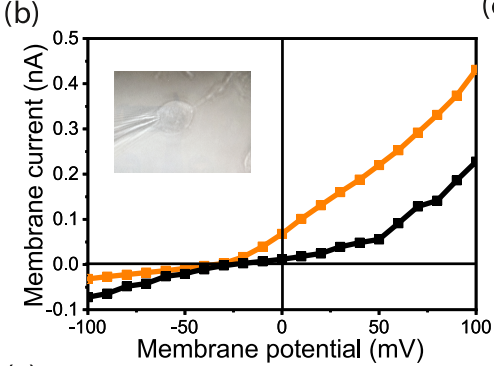

(e)

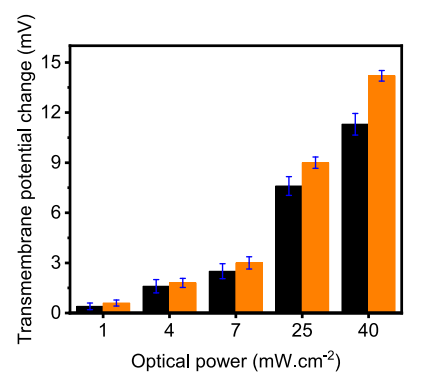

(c)

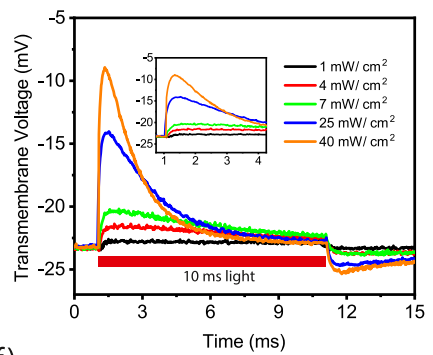

(f)

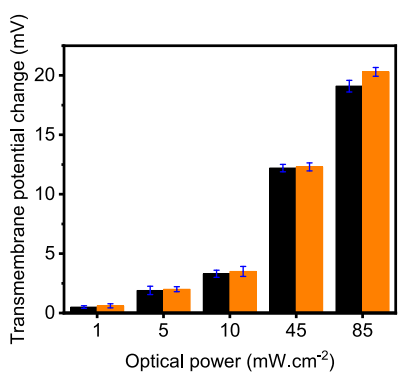

Figure 5. (a) Schematic of the whole-cell patch-clamp recording configuration of the biointerface in wireless and free-standing modes. The cells are grown on the biointerfaces and adhere to the surface. (b) Current-voltage characteristics of a typical SHSY-5Y cell adhered to the control (black line) and plasmonic (orange line) biointerfaces. The inset shows the photograph of a SHSY-5Y cell on the plasmonic biointerface patched with a glass micropipette. (c) Transmembrane potential variation of SHSY-5Y cells on the plasmonic biointerface under green light illumination with 10 ms pulse width. (d-f) Transmembrane potential variation of SHSY-5Y cells on the control (black) and plasmonic (control) biointerfaces under blue (d), green (e), and red (f) light illumination with $10 \mathrm{~ms}$ pulse width. (g) Transmembrane potential under $10 \mathrm{~ms}$ pulse width.

potential for both biointerfaces. Under $10 \mathrm{~ms}$ green light (1$\left.40 \mathrm{~mW} \cdot \mathrm{cm}^{-2}\right)$, an initial depolarization of a single-cell membrane depolarization is followed by a hyperpolarization (Figure 5c). Since the current direction of the first capacitive peak current is from the biointerface toward the substrate in the attached membrane part, the current leads to a depolarization of the free membrane. In contrast, the second peak of the capacitive current leads to a hyperpolarization of the free membrane (see Figure S10). Similar membrane behavior is also observed under green and red illumination as well (Figure S11). Moreover, under repetitive excitation, the peak membrane potential change is also stable under different illumination colors (Figures 5e and S12 and Table S8).

In comparison with the control biointerface, we observed a $37.3 \%$ enhancement in the peak depolarization of the transmembrane potential of the SHSY-5Y cells under blue excitation (Figure 5d). When the excitation is shifted to red color, we observed a lower peak enhancement of $7.7 \%$ (Figure 5e,f, eqs S5 and S6, and Table S7). This shows that the strength of the stimuli due to the nanoantenna effect shows a reduction in comparison with the hot-electron injection. We attribute that since the wave vector orthogonality to the plane of the biointerface is important for the nanoantenna effect (Figures S13 and S14), the refractive index profile change due to the cell membrane and intracellular environment surround- ing the metallic nanoislands possibly disturbs the wave vector of the biointerface ${ }^{29}$ and decreases the enhancement in the red spectral window.

\section{DISCUSSION}

Hot-electron injection is the dominant electronic mechanism for the enhancement of capacitive stimulation. The photons in both the blue and red spectral windows have sufficient energy to generate hot electrons. Since the excited-state energy of hot electrons under blue excitation is higher than that under red excitation, the hot-electron injection to the biointerface pumped under blue illumination is more probable. However, the decrease in the hot-electron injection probability in the red spectral region is compensated by the near-field enhancement of the optical energy in the bulk heterojunction $(\mathrm{BHJ})$ composite.

To capture the hot electrons efficiently, we formed a Schottky barrier with the PTB7-Th:PC71BM bulk heterojunction $(\mathrm{BHJ})$ composite. Since the generated hot electrons with energies higher than the Schottky barrier energy experience lower interfacial resistance toward the $\mathrm{BHJ}$ in comparison with the electrode-electrolyte interface, they can be efficiently transferred to the photoactive layer, instead of donating the electron to the electrolyte for faradic reactions. 
We also observed this fact from the low magnitude of the faradic photocurrents. After the hot electrons are injected into the optoelectronic biointerface, the gold nanoislands are left with holes. Since the capacitive current in the plasmonic biointerface is effectively based on hole accumulation at the electrode-electrolyte interface, the holes remained inside the gold nanoislands after hot-electron injection and the ones that moved to PTB7-Th due to low potential barrier lead to capacitive enhancement. Therefore, even though electron relaxation through electron-electron and electron-phonon collisions that are converted into heat energy was generally used as the main mechanism for cell stimulation, ${ }^{4-8}$ differently in this study, both hot-electron injection and near-field enhancement are used to improve the displacement currents.

The intensity levels that are applied here for cell stimulation can be safely used for all of the tissue or cell types. For example, the most light-sensitive part of the human body is the retina, and the design of the biointerfaces needs to satisfy maximum permissible exposures for ocular safety limits allowed for ophthalmic applications. In this regard, $1 \mathrm{~mW}$. $\mathrm{cm}^{-2}$ light sensitivity of the plasmonic biointerface corresponds to more than three orders of magnitude smaller than ocular safety limits (see the Materials and Methods section). Although the transmembrane potential variation in the free membrane seems low, the variation in the attached membrane is significantly larger; ${ }^{28}$ moreover, the current levels (over 100 pA) that are observed using similar electrophysiological measurements $^{30}$ are enough to elicit action potentials. Therefore, these plasmonic biointerfaces are potentially applicable to recover the vision due to photoreceptor loss in the retina. ${ }^{6}$ This kind of sight loss is frequent in the clinics due to age-macular degeneration, retinitis pigmentosa, and Stargardt's disease. ${ }^{31-33}$

Gold nanoislands have a high potential for biological applications due to their biocompatibility, easy fabrication, and control over optical properties. They have strong absorption from the visible to the NIR region; therefore, plasmon-coupled biointerfaces can lead to a new solution for capacitive stimulation for deep-brain regions. The plasmonic absorption arises due to the collective oscillation of the conduction-band electrons in the resonance peak of the absorbance spectrum, and its resonance peak of the nanometals can be further controlled by alloying with different metals (e.g., silver) or using different shapes (e.g., cube). ${ }^{34}$ Hence, the combination of the plasmonics with optoelectronic biointerfaces offers a wide variety of device architectures for cell stimulation that can operate at different spectral windows for photomedicine.

In this study, we incorporated plasmonics to light-activated capacitive neurostimulators. Hot-electron injection and the nanoantenna effect significantly enhanced the injected charge amounts and currents from the biointerface to the electrolyte. This facilitated variations in the membrane potential at the single-cell level within the low-intensity levels of several $\mathrm{mW}$. $\mathrm{cm}^{-2}$, inside the safe limits for retinal photostimulation. In addition to the safe intensity levels, the capacitive charge injection mechanism is a biocompatible neurostimulation strategy that can be applied for a long term and repeatedly to the targeted neurological cell type. We believe that the combination of plasmonics and optoelectronic biointerfaces points out a bright future for novel nanoengineered optoelectronic neural interfaces.

\section{MATERIALS AND METHODS}

Materials. Poly-[4,8-bis(5-(2-ethylhexyl)thiophen-2-yl)benzo[1,2b;4,5-b'] dithiophene-2,6-diyl-alt-(4-(2-ethylhexyl)-3-fluorothieno[3,4-b]thiophene-)-2-carboxylate-2-6-diyl)] (PTB7-Th) with a molecular weight of $57467 \mathrm{~g} \mathrm{~mol}^{-1}$ and [6,6]-phenyl-C71-butyric acid methyl ester (PC71BM) with a molecular weight of $1031 \mathrm{~g} \mathrm{~mol}^{-1}$ were purchased from Ossila Ltd. and used as received. Commercial gold $(\mathrm{Au})$ with $99.95 \%$ purity was used for plasmonic gold nanoisland fabrication. Other materials and processing chemicals such as zinc acetate dihydrate $\left(\mathrm{Zn}\left(\mathrm{CH}_{3} \mathrm{CO}_{2}\right)_{2} \cdot 2 \mathrm{H}_{2} \mathrm{O}\right)$ with a molecular weight of $183.48 \mathrm{~g} \mathrm{~mol}^{-1}$, ethanolamine $\left(\mathrm{HOCH}_{2} \mathrm{CH}_{2} \mathrm{NH}_{2}\right)$ with a molecular weight of $61.08 \mathrm{~g} \mathrm{~mol}^{-1}$, and 1,2-dichlorobenzene were purchased from Sigma-Aldrich and used without any purification.

Biointerface Preparation. Two types of biointerfaces with and without gold nanoislands (AuNIs) were fabricated. Indium tin oxide (ITO) on glass substrates (side length, $20 \mathrm{~mm}$; side length, $15 \mathrm{~mm}$; thickness, $1.1 \mathrm{~mm}$; resistance, $14-16 \Omega \cdot \mathrm{cm}^{-2}$; Ossila) was cleaned with 10 wt \% sodium hydroxide solution for $5 \mathrm{~min}, 2 \%$ (by volume) of specific tension-active agent in a water solution (HELLMANEX II, $3 \%$ ) for $15 \mathrm{~min}$ at $55^{\circ} \mathrm{C}$, DI water for $15 \mathrm{~min}$, acetone for $15 \mathrm{~min}$, and isopropyl alcohol for $15 \mathrm{~min}$ using an ultrasonic bath. The cleaned ITO on glass-coated substrates was dried in an oven at $50{ }^{\circ} \mathrm{C}$ and treated with UV-ozone for $15 \mathrm{~min}$. The $\mathrm{ZnO}$ precursor sol-gel solution $(0.45 \mathrm{M})$ was prepared by mixing $219.3 \mathrm{mg}$ of zinc acetate dihydrate $\left(\mathrm{Zn}\left(\mathrm{CH}_{3} \mathrm{CO}_{2}\right)_{2} \cdot 2 \mathrm{H}_{2} \mathrm{O}\right), 2 \mathrm{~mL}$ of 2-methoxyethanol $\left(\mathrm{C}_{3} \mathrm{H}_{8} \mathrm{O}_{2}\right)$, and $73 \mathrm{mg}$ of ethanolamine $\left(\mathrm{HOCH}_{2} \mathrm{CH}_{2} \mathrm{NH}_{2}\right)$ and ultrasonicated at $55{ }^{\circ} \mathrm{C}$ for $15 \mathrm{~min} .{ }^{25}$ The donor copolymer PTB7-Th (14.4 $\mathrm{mg} \mathrm{mL}^{-1}$ ) and $21.6 \mathrm{mg} \mathrm{mL}^{-1}$ acceptor molecule PC71BM solution were prepared in o-dichlorobenzene and stirred overnight at $70{ }^{\circ} \mathrm{C}$ on a hot plate. Then, $14.4 \mathrm{mg} \mathrm{mL} \mathrm{m}^{-1}$ PTB7-Th and $21.6 \mathrm{mg}$ $\mathrm{mL}^{-1}$ PC71BM (1:1.5 wt ratio) in o-dichlorobenzene and 3\% (by volume $)$ of 1,8-diiodooctane $\left(\mathrm{C}_{8} \mathrm{H}_{16} \mathrm{I}_{2}\right)$ were added and stirred for $3 \mathrm{~h}$ on a hot plate at $70{ }^{\circ} \mathrm{C}$. The control biointerface was fabricated by spin-coating the $\mathrm{ZnO}$ precursor sol-gel solution at $2000 \mathrm{rpm}$ for $60 \mathrm{~s}$ on a cleaned ITO coated on the glass substrate and annealed on a hot plate at $290{ }^{\circ} \mathrm{C}$ for $15 \mathrm{~min} .{ }^{23}$ Further, on the ITO/ZnO substrate, the photoactive blend of the PTB7-Th/PC71BM (1:1.5 wt ratio) solution in o-dichlorobenzene was spin-coated at $600 \mathrm{rpm}$ for $200 \mathrm{~s}$, then dried under nitrogen flow for $15 \mathrm{~min}$, and heated at $100{ }^{\circ} \mathrm{C}$ for $5 \mathrm{~min}$. The plasmonic biointerface was fabricated on a control biointerface by coating $5 \mathrm{~nm}$ of Au using a thermal evaporator (Bruker, MB Evap) at the rate of $0.02-0.03 \mathrm{~nm} \mathrm{~s}^{-1}$ under $6.0 \times 10^{-6}$ mbar vacuum pressure. Due to the thin layer of gold forming nanoislands even at room temperature, it is annealed at $100{ }^{\circ} \mathrm{C}$, which is the maximum temperature that the photoactive layer can withstand before its optical properties start deteriorating.

Optical and Surface Characterizations. UV-vis transmittance (Edinburg) was used to measure the absorbances of the control and plasmonic biointerfaces. Scanning electron microscopy (SEM, Zeiss) was used for imaging the surface profiles and the cross-sectional structures of the control and plasmonic biointerfaces. For better visualization of the surface, an additional thin gold coating is deposited.

Photocurrent Measurements. Further, the photocurrent measurements were performed on a setup including an Olympus T2 upright microscope. An extracellular patch-clamp (EPC) 800 patchclamp amplifier (HEKA Elektronik) is used for measuring photocapacitive currents. The experiment was carried out at room temperature in an extracellular aCSF aqueous medium as the supporting electrolyte solution. The extracellular medium (aCSF) was prepared by mixing $10 \mathrm{mM}$ of 4-(2-hydroxyethyl)-1-piperazineethanesulfonic acid (HEPES), $10 \mathrm{mM}$ glucose, $2 \mathrm{mM} \mathrm{CaCl}_{2}, 140 \mathrm{mM}$ $\mathrm{NaCl}, 1 \mathrm{mM} \mathrm{MgCl}_{2}$, and $3 \mathrm{mM} \mathrm{KCl}$ in distilled water, and the $\mathrm{pH}$ was calibrated to 7.4 using $1 \mathrm{M} \mathrm{NaOH}$. For light stimulation, a Thorlabs blue LED (M450LP1) with a nominal wavelength of $445 \mathrm{~nm}$ was varied between $165 \mu \mathrm{W} \cdot \mathrm{cm}^{-2}$ and $169 \mathrm{~mW} \cdot \mathrm{cm}^{-2}$, a green LED (M530L3) with a nominal wavelength of $530 \mathrm{~nm}$ was varied between $89 \mu \mathrm{W} \cdot \mathrm{cm}^{-2}$ and $41 \mathrm{~mW} \cdot \mathrm{cm}^{-2}$, and a red LED with a nominal wavelength of $630 \mathrm{~nm}$ was varied between $64 \mu \mathrm{W} \cdot \mathrm{cm}^{-2}$ and $85 \mathrm{~mW}$. 
$\mathrm{cm}^{-2}$ (Figures S2 and S3). All LEDs were driven by a DC2200 HighPower 1-Channel LED Driver with Pulse Modulation (Thorlabs). A power meter (Newport 843-R) was used to measure the exact power of light reaching the surface of the biointerface. The illumination was focused on the water immersion objective holder from the photoactive layer side of the biointerface. The illumination area was $1 \mathrm{~cm}^{2}$. The biointerface corners were cleaned with toluene to stabilize the photocurrent measurements.

Electrochemical Measurements. Electrochemical impedance spectroscopy (EIS) in frequency response analysis (FRA) potential scan mode was performed on an Autolab Potentiostat Galvanostat PGSTAT (Metrhom, Netherlands). A three-electrode setup consisting of $\mathrm{Ag} / \mathrm{AgCl}$ as the reference electrode, a platinum wire as the counter electrode, and the biointerface as the working electrode was used. The experiment was carried out at room temperature in an extracellular aCSF aqueous medium as the supporting electrolyte solution. The biointerfaces were excited by blue, green, and red LEDs with an optical power output of $25 \mathrm{~mW} \cdot \mathrm{cm}^{-2}$. In this mode, a sinusoidal alternating voltage perturbation with a root-mean-square value of $10 \mathrm{mV}$ was applied, while the frequency was varied from $1 \mathrm{~Hz}$ to $0.1 \mathrm{MHz}$. The electrochemical responses were fitted with a proper circuit diagram using NOVA software.

Using the macroelectrical parameters obtained by fitting, the driving electrical parameters in the bulk of the biointerface such as the effective lifetime $\left(\tau_{\mathrm{n}}\right)$ and mobility $\left(\mu_{\mathrm{n}}\right)$ of charge carriers can be estimated using the following equations

$$
\begin{aligned}
& C_{\mu}=\frac{\left(Y_{0} R_{\mathrm{rec}}\right)^{1 / n}}{R_{\mathrm{rec}}} \\
& \tau_{\mathrm{n}}=R_{\mathrm{rec}} C_{\mu} \\
& \tau_{\mathrm{d}}=r_{\mathrm{t}} C_{\mu} \\
& D_{\mathrm{n}}=\frac{L^{2}}{\tau_{\mathrm{d}}} \\
& \mu_{\mathrm{n}}=e D_{\mathrm{n}} / k_{\mathrm{B}} T
\end{aligned}
$$

where $C_{\mu}$ is the chemical capacitance corresponding to carrier accumulation in the bulk, $\tau_{\mathrm{d}}$ is the electron diffusion time, $D_{\mathrm{n}}$ is the diffusion coefficient of electrons, $L$ is the thickness of the photoactive thin film, and $k_{\mathrm{B}} T$ is the thermal energy.

MTT Assay. The cytotoxic effect of biointerfaces on SHSY-5Y cells was assessed by measuring the mitochondrial activity with the MTT assay. The MTT assay was performed as described by Bahmani Jalali et al. Briefly, biointerfaces and the ITO control were sterilized with $70 \%$ ethanol and followed by UV-C treatment for $5 \mathrm{~min}$. Sterilized samples were placed in a six-well cell culture plate. Then, SH-SY5Y with $0.3 \times 10^{6}$ cells were seeded into each well in Dulbecco's modified Eagle medium (DMEM) containing $10 \%$ fetal bovine serum (FBS) and $1 \%$ penicillin-streptomycin. Cell attachment and growth were allowed by $48 \mathrm{~h}$ incubation at $37{ }^{\circ} \mathrm{C}$ and $5 \% \mathrm{CO}_{2}$. After incubation, the growth medium was discarded, and samples were transferred to a new six-well plate. 3-(4,5-Dimethylthiazol-2-yl)-2,5-diphenyltetrazolium bromide (MTT, $1 \mathrm{mg} \mathrm{mL}^{-1}$ ) was added to the samples in serumfree DMEM and incubated for $4 \mathrm{~h}$ at $37{ }^{\circ} \mathrm{C}$ to allow formazan formation. The MTT solution was removed, and formazan salts were dissolved in 50:50 (v:v) ethanol/dimethyl sulfoxide mixture (EtOH/ DMSO). Absorbances of each sample and ITO control were measured in a 96-well plate with a Synergy H1 microplate reader (BioTek). The relative absorbance of biointerface samples to ITO was calculated to determine the relative biocompatibility.

LDH Leakage Assay. The LDH leakage assay (CytoSelect LDH cytotoxicity assay kit, CBA-241, Cell Biolabs) was performed to assess the membrane integrity of the cells grown on the substrates. The experiment was performed according to the manufacturer's manual. Briefly, $3 \times 10^{5}$ SHSY-5Y cells were seeded onto sterilized biointerfaces and control groups as explained in the MTT Assay section. Two sets of the ITO control were included as positive and negative control groups. Samples were incubated at $37{ }^{\circ} \mathrm{C}$ and $5 \%$ $\mathrm{CO}_{2}$ in a time-dependent manner $(24,48$, and $72 \mathrm{~h})$. Negative and positive ITO controls were treated with $1 \%$ Triton X-100 and $\mathrm{dH}_{2} \mathrm{O}$, respectively. The medium $(90 \mu \mathrm{L})$ from each sample was transferred to a 96-well plate and mixed with $10 \mu \mathrm{Lof} \mathrm{LDH}$ cytotoxicity assay reagent. The plate was incubated at $37^{\circ} \mathrm{C}$ for $30 \mathrm{~min}$. The presence of $\mathrm{LDH}$ in the medium was measured by absorbance at $450 \mathrm{~nm}$ with a Synergy H1 microplate reader (BioTek).

Immunolabeling and Fluorescence Microscopy. Substrates were sterilized, and SHSY-5Y cells were seeded on substrates into a six-well plate as explained in the cytotoxicity assessment section. The six-well plate was incubated at $37{ }^{\circ} \mathrm{C}$ and $5 \% \mathrm{CO}_{2}$ for $48 \mathrm{~h}$. Cells grown on the substrates were washed three times with phosphatebuffered saline containing $0.1 \%$ Triton X-100 (PBSt). Cell fixation was performed by $30 \mathrm{~min}$ incubation at $37{ }^{\circ} \mathrm{C}$ in $4 \%$ paraformaldehyde (PFA). Fixed cells were washed three times with PBSt and blocked with PBSt containing 5\% bovine serum albumin (BSA) for $2 \mathrm{~h}$ at RT. The anti- $\beta$ III tubulin (ab78078, Abcam) primary antibody was used for cytoskeleton labeling, and 4',6diamidino-2-phenylindole (DAPI) staining was performed for nucleus visualization. Samples were incubated with 1:250 diluted anti- $\beta$ III tubulin primary antibody for $2 \mathrm{~h}$ and washed three times with PBSt. The Alexa-Fluor-conjugated goat antimouse IgG H\&L (ab150113) secondary antibody (1:1000) and DAPI (1:10000) were diluted in PBSt containing 5\% BSA, and samples were incubated in the mixture for $1 \mathrm{~h}$. Substrates were washed three times with PBSt and mounted onto microscope slides with Mowiol. Imaging was performed with a fluorescence microscope (Zeiss Observer Z1).

Electrophysiology. Electrophysiology measurements were performed with an EPC 800 patch-clamp amplifier (HEKA Elektronik). The biointerfaces were cleaned with $70 \%$ (by volume) ethanol solution and incubated for 2 days in water. The pulled patch pipettes of 8-12 $\mathrm{M} \Omega$ were used to perform the whole-cell patch-clamp experiment. The extracellular medium (aCSF) was prepared as mentioned previously. The internal cellular medium was prepared by mixing $140 \mathrm{mM} \mathrm{KCl}, 2 \mathrm{mM} \mathrm{MgCl} 2,10 \mathrm{mM}$ HEPE, $10 \mathrm{mM}$ ethylene glycol-bis ( $\beta$-aminoethyl ether)- $N, N, N^{\prime}, N^{\prime}$-tetraacetic acid (EGTA), and $2 \mathrm{mM} \mathrm{Mg-ATP}$ in water and its $\mathrm{pH}$ was calibrated to $7.2-7.3$ using $1 \mathrm{M} \mathrm{KOH}$, and patch pipettes were filled with the intracellular solution. A digital camera integrated with an Olympus T2 upright microscope was used to monitor the cells. The whole-cell patched cells were investigated up to $30 \mathrm{~min}$ to avoid damage done by patched pipettes.

Optical Safety Considerations. According to ocular safety standards for ophthalmic applications, the maximum permissible radiant power (MPF) that could enter the pupil chronically or in a single short exposure is calculated. For single-pulse exposures (pulse width of $10 \mathrm{~ms}$ ) at $400-700 \mathrm{~nm}$, the peak limit is governed by the equation $\mathrm{MPF}=6.93 \times 10^{-4} C_{\mathrm{T}} C_{\mathrm{E}} t^{-0.25}$, where, $t=10 \mathrm{~ms}$ (pulse width), $C_{\mathrm{T}}=1$ in the range of $400-700 \mathrm{~nm}$, and $C_{\mathrm{E}}$ is a function of the visual angle $\alpha$. For retinal spot sizes greater than $1.7 \mathrm{~mm}, \alpha=\alpha_{\max }$ $=100 \mathrm{mrad}$ and $C_{\mathrm{E}}=6.67 \times 10^{-3} \alpha^{2}=66.7 \mathrm{~W}$. For $10 \mathrm{~ms}$ pulse width, $\mathrm{MPF}=146.17 \mathrm{~mW}$. For a pupil size with a diameter of $1.7 \mathrm{~mm}$, the MPF per unit area is $\mathrm{MPF}_{\text {per unit area }}=6443 \mathrm{~mW} \cdot \mathrm{cm}^{-2}$.

\section{ASSOCIATED CONTENT}

\section{Supporting Information}

The Supporting Information is available free of charge at https://pubs.acs.org/doi/10.1021/acsami.0c09455.

SEM images of control and plasmonic biointerface, AuNI size distribution, LED's spectrum, optical setup, figure and table of whole photocurrent measured data, thermal investigation, figure and table of electrochemical measurements, figure and table of whole electrophysiology measured data, Lumerical FTDT simulations for different $k$ vectors, and AuNI and control biointerface temperature treatment data (PDF) 


\section{AUTHOR INFORMATION}

\section{Corresponding Author}

Sedat Nizamoglu - Department of Electrical and Electronics Engineering and Graduate School of Biomedical Sciences and Engineering, Koc University, Istanbul 34450, Turkey; ๑ orcid.org/0000-0003-0394-5790; Email: snizamoglu@ ku.edu.tr

\section{Authors}

Rustamzhon Melikov - Department of Electrical and Electronics Engineering, Koc University, Istanbul 34450, Turkey; $\odot$ orcid.org/0000-0003-2214-7604

Shashi Bhushan Srivastava - Department of Electrical and Electronics Engineering, Koc University, Istanbul 34450, Turkey

Onuralp Karatum - Department of Electrical and Electronics Engineering, Koc University, Istanbul 34450, Turkey; ๑ orcid.org/0000-0002-7669-9589

Itir Bakis Dogru-Yuksel - Graduate School of Biomedical Sciences and Engineering, Koc University, Istanbul 34450, Turkey; ๑ orcid.org/0000-0001-8569-7625

Houman Bahmani Jalali - Graduate School of Biomedical Sciences and Engineering, Koc University, Istanbul 34450, Turkey; @ orcid.org/0000-0001-7212-9098

Sadra Sadeghi - Graduate School of Materials Sciences and Engineering, Koc University, Istanbul 34450, Turkey; ๑ orcid.org/0000-0002-8569-1626

Ugur Meric Dikbas - Molecular Biology and Genetics, College of Science, Koc University, Istanbul 34450, Turkey

Burak Ulgut - Department of Chemistry, Bilkent University, Ankara 06800, Turkey; ๑ orcid.org/0000-0002-4402-0033

Ibrahim Halil Kavakli - Molecular Biology and Genetics, College of Science and College of Engineering, Chemical and Biological Engineering, Koc University, Istanbul 34450, Turkey; ๑ orcid.org/0000-0001-6624-3505

Arif E. Cetin - Izmir Biomedicine and Genome Center, Izmir 35330, Turkey

Complete contact information is available at: https://pubs.acs.org/10.1021/acsami.0c09455

\section{Author Contributions}

R.M. and S.B.S. contributed equally. R.M., S.B.S., and S.N. designed the experiments. U.M.D. and I.H.K. performed cell seeding and biological assays. R.M., S.B.S., and O.K. performed the electrophysiology and photocurrent experiments. R.M. and S.B.S. performed the electrochemical measurements. I.B.D.-Y. designed the optical setup. S.S. and H.B.J. helped R.M. for optical measurements. R.M. and A.E.C. performed FDTD simulations. R.M., S.B.S., B.U., and S.N. analyzed the data. R.M., S.B.S., O.K., and S.N. wrote the manuscript, with input from all authors.

\section{Notes}

The authors declare no competing financial interest.

\section{ACKNOWLEDGMENTS}

This project has received funding from the European Research Council (ERC) under the European Union Horizon 2020 Research and Innovation Programme (grant agreement no. 639846). S.N. acknowledges the support from the Turkish Academy of Sciences (TÜBA-GEBiP; The Young Scientist Award Program) and the Science Academy of Turkey (BAGEP; The Young Scientist Award Program). The authors thank Dr. Gokhan Demirel at Gazi University for fruitful discussions.

\section{REFERENCES}

(1) da Cruz, L.; Dorn, J. D.; Humayun, M. S.; Dagnelie, G.; Handa, J.; Barale, P. O.; Sahel, J. A.; Stanga, P. E.; Hafezi, F.; Safran, A. B.; Salzmann, J.; Santos, A.; Birch, D.; Spencer, R.; Cideciyan, A. V.; de Juan, E.; Duncan, J. L.; Eliott, D.; Fawzi, A.; Olmos de Koo, L. C.; Ho, A. C.; Brown, G.; Haller, J.; Regillo, C.; Del Priore, L. V.; Arditi, A.; Greenberg, R. J.; Argus II Study Group. Five-Year Safety and Performance Results from the Argus II Retinal Prosthesis System Clinical Trial. Ophthalmology 2016, 123, 2248-2254.

(2) Yuan, X.; Hierlemann, A.; Frey, U. Dual-mode microelectrode array with 20k-electrodes and high SNR for high-throughput extracellular recording and stimulation. Front. Cell. Neurosci. 2018, 12, No. 88.

(3) Grill, W. M.; Mclntyre, C. C. Extracellular excitation of central neurons: implications for the mechanisms of deep brain stimulation. Thalamus Relat. Syst. 2001, 1, 269-277.

(4) Bareket-Keren, L.; Hanein, Y. Novel interfaces for light directed neuronal stimulation: advances and challenges. Int. J. Nanomed. 2014, 9,65 .

(5) Rand, D.; Jakešová, M.; Lubin, G.; Vèbraitè, I.; David-Pur, M.; Đerek, V.; Cramer, T.; Sariciftci, N. S.; Hanein, Y.; Głowacki, E. D. Direct Electrical Neurostimulation with Organic Pigment Photocapacitors. Adv. Mater. 2018, 30, No. 1707292.

(6) Ghezzi, D.; Antognazza, M. R.; Maccarone, R.; Bellani, S.; Lanzarini, E.; Martino, N.; Mete, M.; Pertile, G.; Bisti, S.; Lanzani, G.; Benfenati, F. A polymer optoelectronic interface restores light sensitivity in blind rat retinas. Nat. Photonics 2013, 7, 400.

(7) Bahmani Jalali, H.; Mohammadi Aria, M.; Dikbas, U. M.; Sadeghi, S.; Ganesh Kumar, B.; Sahin, M.; Kavakli, I. H.; Ow-Yang, C. W.; Nizamoglu, S. Effective neural photostimulation using indiumBased type-II quantum dots. ACS Nano 2018, 12, 8104-8114.

(8) Jiang, Y.; Li, X.; Liu, B.; Yi, J.; Fang, Y.; Shi, F.; Gao, X.; Sudzilovsky, E.; Parameswaran, R.; Koehler, K.; Nair, V.; Yue, J.; Guo, K.; Fang, Y.; Tsai, H. M.; Freyermuth, G.; Wong, R. C. S.; Kao, C. M.; Chen, C. T.; Nicholls, A. W.; Wu, X.; Shepherd, G. M. G.; Tian, B. Rational design of silicon structures for optically controlled multiscale biointerfaces. Nat. Biomed. Eng. 2018, 2, 508-521.

(9) Gilbert, F.; Harris, A. R.; Kapsa, R. M. Controlling brain cells with light: ethical considerations for optogenetic clinical trials. AJOB Neurosci. 2014, 5, 3-11.

(10) Maya-Vetencourt, J. F.; Ghezzi, D.; Antognazza, M. R.; Colombo, E.; Mete, M.; Feyen, P.; Desii, A.; Buschiazzo, A.; Di Paolo, M.; Di Marco, S.; Ticconi, F.; Emionite, L.; Shmal, D.; Marini, C.; Donelli, I.; Freddi, G.; Maccarone, R.; Bisti, S.; Sambuceti, G.; Pertile, G.; Lanzani, G.; Benfenati, F. A fully organic retinal prosthesis restores vision in a rat model of degenerative blindness. Nat. Mater. 2017, 16, 681-689.

(11) Di Maria, F.; Lodola, F.; Zucchetti, E.; Benfenati, F.; Lanzani, G. The evolution of artificial light actuators in living systems: from planar to nanostructured interfaces. Chem. Soc. Rev. 2018, 47, 47574780.

(12) Zimmerman, J. F.; Tian, B. Nongenetic Optical Methods for Measuring and Modulating Neuronal Response. ACS Nano 2018, 12, 4086-4095.

(13) Tatsuma, T.; Nishi, H.; Ishida, T. Plasmon-induced charge separation: chemistry and wide applications. Chem. Sci. 2017, 8, 3325-3337.

(14) Carvalho-de-Souza, J. L.; Treger, J. S.; Dang, B.; Kent, S. B.; Pepperberg, D. R.; Bezanilla, F. Photosensitivity of neurons enabled by cell-targeted gold nanoparticles. Neuron 2015, 86, 207-217.

(15) Eom, K.; Kim, J.; Choi, J. M.; Kang, T.; Chang, J. W.; Byun, K. M.; Jun, S. B.; Kim, S. J. Enhanced infrared neural stimulation using localized surface plasmon resonance of gold nanorods. Small 2014, $10,3853-3857$. 
(16) Yoo, S.; Hong, S.; Choi, Y.; Park, J.-H.; Nam, Y. Photothermal inhibition of neural activity with near-infrared-sensitive nanotransducers. ACS Nano 2014, 8, 8040-8049.

(17) Lee, J. W.; Jung, H.; Cho, H. H.; Lee, J. H.; Nam, Y. Gold nanostar-mediated neural activity control using plasmonic photothermal effects. Biomaterials 2018, 153, 59-69.

(18) Nakatsuji, H.; Numata, T.; Morone, N.; Kaneko, S.; Mori, Y.; Imahori, H.; Murakami, T. Thermosensitive Ion Channel Activation in Single Neuronal Cells by Using Surface-Engineered Plasmonic Nanoparticles. Angew. Chem., Int. Ed. 2015, 54, 11725-11729.

(19) Soganci, I. M.; Nizamoglu, S.; Mutlugun, E.; Akin, O.; Demir, $\mathrm{H}$. V. Localized plasmon-engineered spontaneous emission of CdSe/ $\mathrm{ZnS}$ nanocrystals closely-packed in the proximity of $\mathrm{Ag}$ nanoisland films for controlling emission linewidth, peak, and intensity. Opt. Express 2007, 15, 14289-14298.

(20) Hoang, C. V.; Hayashi, K.; Ito, Y.; Gorai, N.; Allison, G.; Shi, X.; Sun, Q.; Cheng, Z.; Ueno, K.; Goda, K.; Misawa, H. Interplay of hot electrons from localized and propagating plasmons. Nat. Commun. 2017, 8, No. 771.

(21) Carey, J. D.; Ong, L.; Silva, S. R. P. Formation of lowtemperature self-organized nanoscale nickel metal islands. Nanotechnology 2003, 14, 1223.

(22) Zhao, L.; Zhao, S.; Xu, Z.; Qiao, B.; Huang, D.; Xu, X. Two effects of 1, 8-diiodooctane on PTB7-Th:PC71BM polymer solar cells. Org. Electron. 2016, 34, 188-192.

(23) Srivastava, S. B.; Melikov, R.; Aria, M. M.; Dikbas, U. M.; Kavakli, I. H.; Nizamoglu, S. Band Alignment Engineers Faradic and Capacitive Photostimulation of Neurons Without Surface Modification. Phys. Rev. Appl. 2019, 11, No. 044012.

(24) Bhattacharya, G.; Sankaran, K. J.; Srivastava, S. B.; Thomas, J. P.; Deshmukh, S.; Pobedinskas, P.; Singh, S. P.; Leung, K. T.; Van Bael, M. K.; Haenen, K.; Roy, S. S. Probing the flat band potential and effective electronic carrier density in vertically aligned nitrogen doped diamond nanorods via electrochemical method. Electrochim. Acta 2017, 246, 68-74.

(25) Srivastava, S. B.; Sonar, P.; Singh, S. P. Charge transport studies in donor-acceptor block copolymer PDPP-TNT and PC71BM based inverted organic photovoltaic devices processed in room conditions. AIP Adv. 2015, 5, No. 077177.

(26) Maurano, A.; Hamilton, R.; Shuttle, C. G.; Ballantyne, A. M.; Nelson, J.; O'regan, B.; Zhang, W.; McCulloch, I.; Azimi, H.; Morana, M.; et al. Recombination dynamics as a key determinant of open circuit voltage in organic bulk heterojunction solar cells: a comparison of four different donor polymers. Adv. Mater. 2010, 22, 4987-4992.

(27) Abdullaeva, O. S.; Balzer, F.; Schulz, M.; Parisi, J.; Lützen, A.; Dedek, K.; Schiek, M. Organic Photovoltaic Sensors for Photocapacitive Stimulation of Voltage-Gated Ion Channels in Neuroblastoma Cells. Adv. Funct. Mater. 2019, 29, No. 1805177.

(28) Jakešová, M.; Ejneby, M. S.; Derek, V.; Schmidt, T.; Gryszel, M.; Brask, J.; Schindl, R.; Simon, D. T.; Berggren, M.; Elinder, F. Optoelectronic control of single cells using organic photocapacitors. Sci. Adv. 2019, 5, No. eaav5265.

(29) Gather, M. C.; Yun, S. H. Single-cell biological lasers. Nat. Photonics 2011, 5, 406.

(30) Parameswaran, R.; Carvalho-de-Souza, J. L.; Jiang, Y.; Burke, M. J.; Zimmerman, J. F.; Koehler, K.; Phillips, A. W.; Yi, J.; Adams, E. J.; Bezanilla, F. Photoelectrochemical modulation of neuronal activity with free-standing coaxial silicon nanowires. Nat. Nanotechnol. 2018, 13, 260 .

(31) Bressler, N. M.; Bressler, S. B.; Fine, S. L. Age-related macular degeneration. Surv. Ophthalmol. 1988, 32, 375-413.

(32) Hartong, D. T.; Berson, E. L.; Dryja, T. P. Retinitis pigmentosa. Lancet 2006, 368, 1795-1809.

(33) Delori, F. C.; Staurenghi, G.; Arend, O.; Dorey, C. K.; Goger, D. G.; Weiter, J. J. In vivo measurement of lipofuscin in Stargardt's disease-Fundus flavimaculatus. Invest. Ophthalmol. Vis. Sci. 1995, 36, 2327-2331.

(34) Sau, T. K.; Rogach, A. L. Complex-Shaped Metal Nanoparticles; Wiley Online Library, 2012. 